\title{
Pain, Catastrophizing, and Depression in Chronic Prostatitis/ Chronic Pelvic Pain Syndrome
}

\author{
Jong Kyou Kwon, In Ho Chang \\ Department of Urology, Chung-Ang University College of Medicine, Seoul, Korea
}

\begin{abstract}
Persistent and disabling pain is the hallmark of chronic prostatitis/chronic pelvic pain syndrome (CP/CPPS). However, disease severity (as measured by objective indexes such as those that use radiography or serology) is only marginally related to patients' reports of pain severity, and pain-related presentation can differ widely among individuals with CP/CPPS. Increasing evidence in support of the biopsychosocial model of pain suggests that cognitive and emotional processes are crucial contributors to inter-individual differences in the perception and impact of pain. This review describes the growing body of literature relating depression and catastrophizing to the experience of pain and pain-related sequelae in CP/CPPS. Depression and catastrophizing are consistently associated with the reported severity of pain, sensitivity to pain, physical disability, poor treatment outcomes, and inflammatory disease activity and potentially with early mortality. A variety of pathways, from cognitive to behavioral to neurophysiological, seem to mediate these deleterious effects. Collectively, depression and catastrophizing are critically important variables in understanding the experience of pain in patients with CP/CPPS. Pain, depression, and catastrophizing might all be uniquely important therapeutic targets in the multimodal management of a range of such conditions.
\end{abstract}

Keywords: Pain; Catastrophization; Depression; Prostatitis

\section{INTRODUCTION}

Chronic prostatitis/chronic pelvic pain syndrome (CP/CPPS) is a common male chronic pain condition. Its prevalence ranges from $9 \%$ to $16 \%$ worldwide and from $5 \%$ to $25 \%$ in Korea [1-3]. $\mathrm{CP} / \mathrm{CPPS}$ presents with various voiding symptoms, perineal or suprapubic pain, erectile dysfunction or sexual disturbance, and psychological problems such as depression and catastrophizing, which have a negative impact on health-related quality of life (QoL) [4,5]. However, the etiology of CP/CPPS is uncertain. Various theories have been hypothesized, such as inflammation, pathogen and host-specific factors, pelvic floor tension myalgia, and differences in systemic pressure sensitivity [6-8]. It has been suggested that this condition has significant psychological components, because most CP/CPPS patients have depression and catastrophizing [9]. It has also been suggested that stress is a potent factor in the development, prolongation, and perpetuation of the symptoms in the condition known generally as chronic prostatitis [10].

The severity of stress has been reported to depend on individual perception or subjective interpretation of causative factors rather than on the contents or frequency of the factors causing stress [11]. That is, individual coping strategies for each stress and cognitive assessment of stress have been suggested to significantly affect stress progression [12]. Coping strategies refer to cognitive and behavioral efforts that are used to satisfy external or internal demands, and they have been known to act as regulators between stress and psychological disorders or physical diseases [13]. Therefore, in addition to stress frequency, differences in stress perception and coping strategies should be considered as factors causing or deteriorating stress symptoms in patients with $\mathrm{CP} / \mathrm{CPPS}$.
Corresponding author: In Ho Chang

Department of Urology, Chung-Ang University Hospital, Chung-Ang University College of Medicine, 102 Heukseok-ro, Dongjak-gu, Seoul 156-755, Korea Tel: +82-2-6299-1819 / Fax: +82-2-6299-1406 / E-mail: caucih@cau.ac.kr Submitted: May 2, 2013 / Accepted after revision: June 15, 2013
This is an Open Access article distributed under the terms of the Creative Commons Attribution Non-Commercial License (http://creativecommons.org/licenses/by-nc/3.0/) which permits unrestricted non-commercial use, distribution, and reproduction in any medium, provided the original work is properly cited. 
Physical disease and psychiatric disorders coexist in CP/CPPS [14], with as many as $78 \%$ of patients with CP/CPPS reporting depression [15] and 60\% meeting the criteria for a major depressive disorder [16]. Furthermore, greater depression and not having a partner for support were associated with poorer $\mathrm{CP} /$ CPPS outcomes [5]. Adding to the depression concern, CP/CPPS pain and QoL outcomes are predicted by a particular set of psychosocial risk factors, including low social support and pain catastrophizing $[5,17,18]$. Pain catastrophizing is associated with chronic pain and it may be a key component in the clinical phenotypic classification of male urologic chronic pelvic pain $[19,20]$. Catastrophizing is the tendency to employ a set of painassociated cognitive appraisals referred to as ruminative ("can't keep it out of my mind"), magnifying ("makes me think about other pains"), and helpless ("there is nothing I can do") when undergoing or anticipating pain [21]. Catastrophizing is associated with anxiety and depression but is considered a unique factor in pain [18,21]. With control for urinary symptoms and depression, catastrophizing was the strongest biopsychosocial predictor of CP/CPPS pain and was shown to be a robust predictor of diminished mental status QoL in men with CP/CPPS $[17,18]$. CP/CPPS research supports the necessity and rationale for a specific cognitive behavioral treatment model. There has been interest in considering psychosocial treatments $[18,22]$ and approaches to reduce the psychosocial risk factors associated with poor CP/CPPS outcomes [23]. Although the concept of "psychogenic" pain has (appropriately) been abandoned, it is clear that negative emotional processes such as depression and catastrophizing can increase the level of pain perceived by patients with $\mathrm{CP} / \mathrm{CPPS}$ through a range of mechanisms, which we review here.

\section{LINKING EMOTIONAL PROCESSES AND PAIN IN CP/CPPS}

A number of small cohort studies have investigated the impact of CP/CPPS on men with regard to QoL, sexual function, and psychological aspects (Table 1) $[5,16]$. In the National Institutes of Health (NIH) Chronic Prostatitis Cohort study, McNaughton Collins et al. [24] reported an association of reduction in mental and physical items of the Short Form-12 QoL measures with increasing severities of CP/CPPS symptoms. Another study, in a large healthy population, indicated that bladder symptoms, including urinary frequency and incomplete emptying, have a greater detrimental effect on QoL than do pain symptoms. A further study, which evaluated the management of CP/CPPS patients in primary care settings, showed that worse QoL is associated with greater pain and urinary symptoms and that pelvic pain is associated with worse QoL than urinary symptoms [25]. On the basis of the results from the 463 men enrolled in the NIH Chronic Prostatitis Cohort Study, which included data from seven clinical centers, Tripp et al. [5] indicated that the severity of pelvic pain can be strongly predicted by depressive symptoms, beyond the contributions of urinary symptom scores and age. Recently, a case-control study conducted by Clemens et al. [26] also showed that depression and panic disorder are significantly more common in men with CP/CPPS than in controls. Many patients with pelvic pain reported continuing symptoms of depression and anxiety despite receiving treatment medications. These findings suggest that the mental health disorders may be more difficult to treat in patients with urologic pain syndromes [26]. Pelvic pain and urinary symptoms both contribute to reduced QoL and depressive mood experienced by CP/CPPS patients [27].

The first study examining catastrophizing in $\mathrm{CP} / \mathrm{CPPS}$ was conducted by the NIH Chronic Prostatitis Collaborative Research Network (CPCRN) Study Group [18,28], which found that catastrophizing was correlated with greater disability, higher urinary scores, depressive symptoms, and pain. Furthermore, catastrophizing was a significant predictor of pain when the analysis was controlled for demographic and psychosocial variables. Another recent study showed that diminished mental status QoL was predicted by greater helplessness catastrophizing and lower social support from friends and family, beyond the demographic, medical status, and other psychosocial variables in the analyses [17]. Furthermore, Tripp et al. [29] showed that an adolescent male sample (aged 16 to 19 years) reported a prevalence of at least mild prostatitis-like symptoms at $8.3 \%$, with $3 \%$ reporting a pain domain score of $>7$.

Pain, urinary symptoms, depressive symptoms, and catastrophizing were all correlated with poorer QoL and the catastrophizing magnification domain was the lone predictor of poorer QoL after control for pain and urinary scores. These findings have increased the desire to model treatment of urologic chronic pelvic pain syndromes from a framework that manages individual patients through the use of a multimodal therapy approach. UPOINT (urological, psychosocial, organ-specific, infection, neurological, and tenderness of skeletal muscles) is a 6-point clinical classification system that includes a psychosocial domain (i.e., catastrophizing) [30]. 
Table 1. Examples of adverse outcomes of depression and catastrophizing in studies

\begin{tabular}{|c|c|c|}
\hline Sample & Outcome & Study \\
\hline \multicolumn{3}{|l|}{ Depression } \\
\hline Patients with CP/CPPS & $\begin{array}{l}\text { Prostatitis patients were employed but reported that their symptoms greatly interfered } \\
\text { with their sexual/romantic relationships, and half of the prostatitis patients met } \\
\text { criteria for major depression }\end{array}$ & $\begin{array}{l}\text { Egan and Krieger (1994) } \\
{[16]}\end{array}$ \\
\hline Patients with $\mathrm{CP} / \mathrm{CPPS}$ & $\begin{array}{l}\text { An association of reduction in mental and physical items of the Short Form-12 QoL } \\
\text { measures with increasing severities of CP/CPPS symptoms }\end{array}$ & $\begin{array}{l}\text { McNaughton Collins et al. } \\
\text { (2001) [24] }\end{array}$ \\
\hline Patients with CP/CPPS & $\begin{array}{l}\text { The severity of pelvic pain can be strongly predicted by depressive symptoms, beyond } \\
\text { the contributions of urinary symptom scores and age }\end{array}$ & Tripp et al. (2004) [5] \\
\hline Case control study & $\begin{array}{l}\text { Depression and panic disorder are significantly more common in men with CP/CPPS } \\
\text { than in controls }\end{array}$ & Clemens et al. (2008) [26] \\
\hline Population-based study & $\begin{array}{l}\mathrm{CP} \text { patients experienced an increased risk of depressive disorder compared with } \\
\text { non-CP patients during a 3-year follow-up period }\end{array}$ & Chung et al. (2011) [7] \\
\hline Cohort study & $\mathrm{CP} / \mathrm{CPPS}$ patients had depression, anxiety, and higher perception of stress & Ahn et al. (2012) [28] \\
\hline \multicolumn{3}{|l|}{ Catastrophizing } \\
\hline Patients with $\mathrm{CP} / \mathrm{CPPS}$ & $\begin{array}{l}\text { Catastrophizing was a significant pain predictor when demographic and psychosocial } \\
\text { variables were controlled }\end{array}$ & Tripp et al. (2006) [18] \\
\hline Patients with CP/CPPS & $\begin{array}{l}\text { Diminished mental status QoL was predicted by greater helplessness catastrophizing } \\
\text { and lower social support from friends and family, beyond the demographic, } \\
\text { medical status, and other psychosocial variables in the analyses }\end{array}$ & Nickel et al. (2008) [17] \\
\hline Adolescent male & $\begin{array}{l}\text { Adolescent male sample (aged } 16 \text { to } 19 \text { years) reported a prevalence of at least mild } \\
\text { prostatitis-like symptoms at } 8.3 \% \text {, with } 3 \% \text { reporting a pain domain score of }>7 \text {. } \\
\text { Catastrophizing was correlated with diminished QoL }\end{array}$ & Tripp et al. (2009) [29] \\
\hline
\end{tabular}

$\mathrm{CP} / \mathrm{CPPS}$, chronic prostatitis/chronic pelvic pain syndrome; QoL, quality of life.

\section{CHANGING PARADIGMS FOR CP/CPPS}

Acute (category I) and chronic (category II) bacterial prostatitis are the best understood and least common of the prostatitis syndromes. CP/CPPS, with or without inflammation, is the third category of prostatitis syndromes. In CP/CPPS type IIIA (with inflammation), urine, semen, and other fluids from the prostate show no evidence of infection. Type IIIB (no inflammation) is regarded as the most common but also the least understood [31]. The fourth category of prostatitis is asymptomatic inflammatory prostatitis in which white blood cells are present in the semen but there is no pain.

\section{A Symptom Complex Rather Than a Specific Disease}

$\mathrm{CP} / \mathrm{CPPS}$ symptoms can vary without warning, with most men reporting acute pain attacks, longstanding persistent pain, or a combination [32]. CP/CPPS pain is localized to urogenital regions (perineum, pelvic area, and/or genitalia) [33,34]. Similar to other chronically painful conditions, the pain in CP/CPPS does not correspond strongly with medical findings [35]. Al- though $\mathrm{CP} / \mathrm{CPPS}$ patients may include those with inflammatory prostate findings, no pathognomonic, histologic, or radiologic findings exist for confirmatory diagnosis. The important reality is that no postulated molecular mechanism explains the symptoms of CP/CPPS. Several case series show lack of CP/CPPS symptom improvement after end organ removal (cystectomy and prostatectomy) $[36,37]$. Thus, CP/CPPS is a symptom complex rather than a specific disease $[38,39]$.

The NIH definition of CP/CPPS is pelvic pain for 3 of the previous 6 months, with or without voiding symptoms and with no evidence of uropathogenic bacterial infection [32]. Symptoms of CP/CPPS do not routinely remit, with $66 \%$ of subjects in community-based samples experiencing symptoms 1 year later [32], and tertiary care patients showing no drop in their pain, depression, or catastrophizing over a multi-year assessment [40]. Treatment successes in CP/CPPS have been described as dismal, with strategies based on sequential monotherapies considered suboptimal for patients with long histories of severe CP/CPPS [31]. The QoL of individuals with CP/CPPS is diminished to a level comparable to that experienced by pa- 
tients with severe congestive heart failure, myocardial infarction, unstable angina, hemodialysis-dependent end-stage renal disease, or active Crohn's disease [24,41].

Thus, CP/CPPS is a heterogeneous condition in terms of its clinical manifestation and, most likely, its underlying mechanism. This heterogeneity is evidenced by four critical observations: 1) the clinical presentation of patients varies from one patient to the next, 2) a single patient may have symptoms that fluctuate over time [42], 3) varying etiological mechanisms (e.g., infection, inflammation, and nerve damage) may account for the observed pathology in the same patient and between patients [43], and 4) the symptoms of CP/CPPS overlap with those of irritable bowel syndrome, fibromyalgia, chronic fatigue syndrome, and other chronic pain disorders [44].

\section{Mechanistic Insight Into CP/CPPS Through Phenotyping}

There is a need for the development of a deeper mechanistic understanding of the etiology and progression of CP/CPPS [45]. Phenotyping is one way to address the knowledge deficit that exists and will improve mechanistic understanding of the syndrome. Phenotyping research explores the etiology and mechanisms of disease and fluctuations in symptoms and pathology over time [46]. A phenotyping system provides a platform through which associations can be made between genotypes and phenotypes. To make these associations, there must be a system for consistently defining and characterizing phenotypes. Thus, new genetic findings can then be placed into a preexisting system and matched and associated with specific clinical presentations.

\section{UPOINT: evaluation of a novel CP/CPPS phenotyping system}

In 2009, Nickel and Shoskes [47] introduced UPOINT, an original system that categorizes CP/CPPS symptoms in six domains: urological, psychosocial, organ-specific, infection, neurological, and tenderness of skeletal muscles. It accords broadly with the NIH Chronic Prostatitis Symptom Index (NIH-CPSI) scale, which currently serves as the standardized symptom severity questionnaire in CP/CPPS. However, it is important to note that CP/CPPS, by definition, is a chronic pain syndrome and therefore is encapsulated by the pain domain $[44,48]$. Thus, a high total NIH-CPSI score may not necessarily reflect pain severity as captured by a high pain-domain score but could result from a high voiding score (representing voiding dysfunction). In such a case, UPOINT is not a sensitive measure of chronic pain symptoms, a hallmark that defines CP/CPPS. Besides, the concordance of UPOINT domains with the NIH-CPSI score is most likely a byproduct of the similarities in scope and approach between the two systems. UPOINT was created on the basis of studies conducted with the use of the NIH-CPSI.

In reality, the UPOINT system emerged because clinical trials (evaluating $\alpha$-blockers, pentosan polysulfate, and pregabalin) failed to detect a significant difference between intervention and placebo, but significant differences were observed in secondary outcomes, such as QoL $[44,47]$. Therefore, it appeared feasible to define cohorts on the basis of secondary outcomes and to establish treatment guidelines for each group. However, the unknown optimal duration of therapy and the placebo effect limit the generalizability of these results [19]. Importantly, UPOINT is limited by the strength of correlation between symptoms and disease mechanisms and provides little improvement in mechanistically driven treatment outcomes when compared with the NIH-CPSI. Lastly, because a single physician examined and diagnosed all the patients, the UPOINT study has the risk of having a biased cohort. Commendably, however, UPOINT recognizes and seeks to address the heterogeneity present in patients with $\mathrm{CP} / \mathrm{CPPS}$.

\section{DABBEC: moving toward a mechanistically predictive phenotyp- ing system}

Allsop et al. [49] proposed the DABBEC (dimitrakoff, allsop, brook, bhai, erstad, and cohen) phenotyping system (DPS) as a clinically useful method for phenotyping patients, designing clinical trials, and evaluating the efficacy of mechanistically driven treatments for CP/CPPS. This system incorporates a view of CP/CPPS as both a localized and a systemic condition. The DPS consists of four scoring domains: the NIH-CPSI, localizedversus-systemic, hypothalamic-pituitary-adrenal axis/sympathetic nervous system (HPA/SNA), and CYP21a2 phenotype.

Following a clinical diagnosis of CP/CPPS, as recommended by the CPCRN guidelines [50], the authors suggest that clinicians administer questionnaire instruments to assess the patient's constitutional, psychological, and cognitive symptom domains. This symptom domain phenotyping will be helpful in identifying comorbidities, such as depression, anxiety, and catastrophizing conditions that are highly prevalent in patients with $\mathrm{CP}$ / CPPS. CPCRN recommends that such patients receive multimodal therapy in the context of multidisciplinary teams of psychologists, neurologists, and internists. 


\section{Psychosocial Domain of UPOINT}

The following psychosocial questionnaires may be useful if it is believed that psychosocial parameters may be important on the basis of probing questions asked during the history taking.

\section{Patient health questionnaire}

This self-administered tool provides an assessment of depression and anxiety [51]. We summed the responses on the depressive symptoms section to create a depressive symptoms index of $0-27$, with higher scores representing greater depressive symptoms.

\section{Pain catastrophizing scale (PCS)}

This scale assesses three measures of negative thoughts associated with pain: rumination or worry, magnification, and helplessness [52]. Rumination and magnification tend to be considered reactionary or proximal cognitive responses to pain experience, whereas helplessness may develop at a later time following ruminative or magnifying thoughts about pain. Helplessness is associated with feelings of despair and represents the internal belief that one cannot manage one's pain. The PCS is reliable [53], and catastrophizing has been shown to be a significant psychological predictor of both pain [18] and QoL [17] for patients with CP/CPPS.

Patients with chronic pain often have maladaptive coping mechanisms (for example, catastrophizing) and may suffer from depression, anxiety, or stress or have a history of sexual or other physical abuse. Psychometric evaluations of men with CP/CPPS compared with healthy control men showed significantly higher scores on all assessments except type A behavior. Especially notable were the highest differential rankings for somatization, depression, psychoticism, and phobic anxiety in men with $\mathrm{CP} /$ CPPS [54]. These observations of psychological disturbances in men with CP/CPPS were anticipated and in agreement with earlier reports [10,18,55-58].

\section{ADVERSE OUTCOMES OF EMOTIONAL PROCESSES}

\section{Increasing Pain Severity and Sensitivity}

Catastrophizing is a robust pain predictor across clinical and nonclinical samples [18] and is important in understanding $\mathrm{CP} / \mathrm{CPPS}$ outcomes. The first study examining catastrophizing in CP/CPPS found it to be associated with greater disability, depression, urinary symptoms, and pain [18]. Furthermore, help- lessness catastrophizing was the strongest pain predictor, even when urinary symptoms and depression were controlled for. In another study, CP/CPPS QoL determinants showed that diminished mental health status was predicted by greater helplessness catastrophizing and lower social support from friends and family, beyond all demographic, medical status, and other competing psychosocial variables [24]. Helplessness catastrophizing is a predominant pain and QoL predictor in CP/CPPS and is expressed by patients reporting longer pain durations (e.g., 4-7 years) [59]. It is worth noting that the relationship between helplessness and pain is not specific to CP/CPPS and is likely to be a manifestation of suffering from long-term pain. Thus, it might be expected that those suffering in the earlier stages of chronicity may manifest different interrelating factors. For example, the male adolescent community-dwelling sample in Canada (16-19 years old) reported a high prevalence of chronic prostatitis-like symptoms (8.3\%) [29], with pain, urinary symptoms, depressive symptoms, and catastrophizing all being associated with diminished QoL. Ancillary analyses also indicated that the magnification subscale for catastrophizing (e.g." "I keep thinking of other painful events") was the lone predictor of poorer QoL when the analysis was controlled for urinary status and pain.

\section{Enhanced Inflammatory Disease Activity}

One experimental study also confirmed that chronic stress in rats can specifically induce histological inflammation of the prostate [60]. In patients with $\mathrm{CP} / \mathrm{CPPS}$ there appear to be some direct, measurable effects of stress or stress decreases on cytokine levels. Chronic activation of the physiologic stress response augments the release of proinflammatory cytokines and prostaglandins that may contribute to CP/CPPS [61]. In CP/CPPS patients, the degree of spousal concern and support as well as an effort to distract the patient from symptoms correlates with lower seminal plasma interleukin (IL)-6 and IL-10 concentrations [62]. Also, patients with depression have been shown to have lower levels of IL-10 in peripheral blood mononuclear cells than do control subjects [63]. Thus, stress changes appear to have measurable effects on proinflammatory cytokine levels in patients with $\mathrm{CP} / \mathrm{CPPS}$, which suggests that there may be a link with histologic inflammation of the prostate [64].

\section{PATHWAYS OF ACTION}

The mechanisms underlying the association of psychosocial 


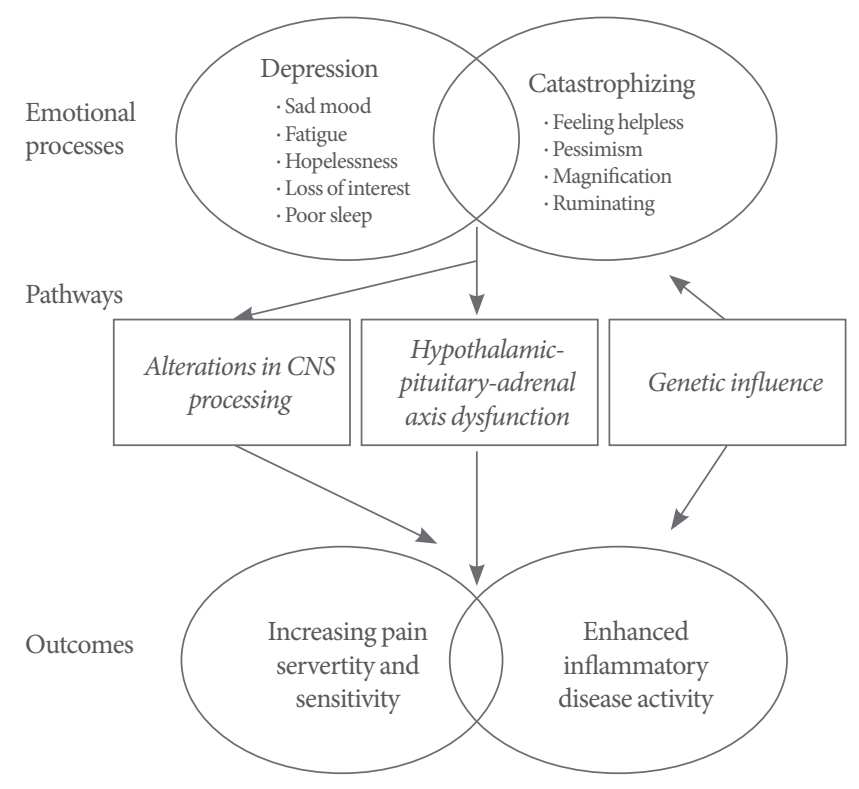

Fig. 1. Depression and catastrophizing: influence on pain-related outcomes. Depression and catastrophizing comprise overlapping yet distinct sets of negative emotional processes. These factors influence a broad spectrum of pain-related outcomes via a variety of pathways. CNS, central nervous system.

factors with CP/CPPS remain unclear. It was suggested that stress accompanied by depression and catastrophizing is a potent factor in the development, prolongation, and perpetuation of CP/CPPS symptoms [10,28]. For the sake of clarify, Fig. 1 simplifies the presentation of these factors as independent factors and the pathways as unidirectional, but the discussion below demonstrates our increasing knowledge of the interdependencies of these mechanisms.

\section{Alterations in Central Nervous System (CNS) Processing}

Incoming signals in the CNS are subject to modulation (both facilitation and inhibition) at a variety of sites. One of the mechanisms by which catastrophizing and depression are thought to influence the experience of pain is by promoting sensitization or by interfering with endogenous pain inhibition in the CNS [65], a hypothesis that can be readily investigated by using noninvasive functional neuroimaging methods such as functional magnetic resonance imaging (fMRI). Among patients with fibromyalgia, for example, those who catastrophized most showed the greatest extent of activation in pain-processing brain areas such as the anterior cingulate and dorsolateral prefrontal cortex during the administration of calibrated noxious stimuli [66]. A recent fMRI study revealed specific pain-related brain activation in patients with CP/CPPS, thus suggesting an ongoing reorganization of the brain circuitry [67]. In a related fMRI study of individuals with major depression, catastrophizing cognitions such as perceived helplessness were related to stronger activation of the amygdala (a key component of the limbic system) during both pain and anticipation of pain [68]. Finally, inducing a depressed mood state increased subjects' level of catastrophizing, impaired their emotion-regulation neurocircuitry, and amplified their cortical responses to a noxious stimulus [69].

Taken together, these functional neuroimaging studies highlight the roles of negative cognitive and emotional processes in amplifying the transmission of pain-related signals in the cortex. Interestingly, one study showed that CP/CPPS is associated with a reduction of relative gray matter in the anterior cingulate cortex [70]. Assuming a core function in pain suppression, the relative reduction in gray matter volume provides evidence of gray matter changes as sequelae of chronic neuroanatomical pain susceptibility. Such evidence supports the findings of corresponding publications regarding chronic back pain, phantom pain, migraine, fibromyalgia, and irritable bowel syndrome [7173]. Moreover, these structural changes in the brain may be most closely associated with depression and emotional aspects of chronic pain $[74,75]$.

We believe that advanced imaging methods, such as brain fMRI and neuroimaging, can provide a unique bridge to disease staging in CP/CPPS. Establishing a universal staging system will provide a uniform language and system of communication among different working groups in terms of the natural history of the disease, progression, and treatment outcomes in clinical trials of CP/CPPS treatments.

\section{HPA Axis Dysfunction}

Depression and catastrophizing can also influence HPA axis responses. Studies have shown that HPA dysregulation may lead to abnormalities of inflammatory responses, resulting in chronic inflammatory and pain conditions such as interstitial cystitis or CP/CPPS $[58,76]$. Men with CP/CPPS have a greater slope of the awakening cortisol response than do age-matched healthy controls, which suggests a dysfunctional HPA axis [58]. The awakening cortisol response serves as a useful index of adrenocortical activity, which provides important information on the activation of the HPA axis. The relatively high intraindividual stability of the awakening cortisol response when measured over repeated days partly reflects a personal trait. However, HPA axis responses can be influenced by environmental factors in- 
cluding depression and catastrophizing and can serve as an indicator of allostatic load. Allostatic load can take the form of alteration in diurnal cortisol rhythm. Systemic cortisol modulates several systems including the balance between cellular and humoral immunity, or the HPA feedback system. HPA axis dysregulation can be manifested either as pathologically decreased or increased cortisol responses. Some abnormalities may lead to dysregulation of inflammatory responses, resulting in chronic inflammatory and pain conditions such as fibromyalgia and interstitial cystitis [76,77]. These symptomatically varied conditions share a common feature of lowered activity of the HPA axis. Blunted awakening cortisol responses are also reported in subjects with burnout or persistent sciatic pain after discectomy $[78,79]$. These conditions are the opposite of the augmented awakening cortisol response that we have observed in patients with CPPS. Several other studies have also shown an association between increased cortisol levels after awakening and psychological variables in chronically stressed individuals owing to work overload $[80,81]$.

Depression and catastrophizing appear to have a similar pattern of HPA axis dysregulation. Whereas hypercortisolism is associated with clinical depression, this observation has also been made in the nonclinical range of depression in healthy young men [82]. The consistent observation that hypercortisolism and depression appear together has led many authors to suggest that increased cortisol levels can cause depressive symptoms and that depressive symptoms can be reversed with antidepressant actions on HPA regulation [82]. Psychological stress has long been implicated as contributory to the etiology or exacerbation of CP/CPPS $[2,10]$. Prospective studies have associated perceived stress longitudinally with pain intensity and have examined cognitive/behavioral variables, such as catastrophizing, as predictors of greater pain intensity and disability [18].

\section{Genetic Influence}

CP/CPPS and depression and catastrophizing may also originate from a shared genetic susceptibility. Among all categories of depression and catastrophizing, panic disorder was mentioned as being highly associated with CP/CPPS. Some prior studies documented that patients with panic disorder were more likely to have symptoms of bladder pain syndrome/interstitial cystitis (BPS/IC) [83,84], which may represent the same underlying condition as CP/CPPS [26]. The possible common genetic susceptibility shared by BPS/IC and depression and catastrophizing might be linked to the Barrington nucleus, which provides an anatomical substrate for coregulation of pelvic visceral symptoms and mental activity in the prosencephalon [85]. Depression and catastrophizing and panic disorder are often comorbid with one another owing to the similarity of their causes, meaning that those who suffer from one often suffer from the other, or will later in life. Therefore, despite the lack of genetic data regarding the association between CP/CPPS and depression and catastrophizing, a common genetic vulnerability might be one possible explanation.

Several studies hint at a potential genetic underpinning for some of the overlap between pain, depression, and catastrophizing. Recent work has focused on the gene coding for catechol $O$-methyltransferase (COMT), an enzyme that degrades catecholamines (which in turn affect the functioning of endogenous opioid systems). To date, certain COMT genotypes have been associated with widespread pain [86], depression [87], and enhanced sensitivity to pain [88]. A daily diary study in patients with fibromyalgia reported a significant interaction between COMT genotype and pain fluctuations, such that individuals with the "high pain sensitivity" genotype experienced a greater decline in positive emotion on days when pain was elevated than did individuals with other COMT genotypes. Similarly, in a prospective study of joint pain [89], COMT genotype interacted with individual differences in catastrophizing to predict longterm pain outcomes. Subjects with high levels of catastrophizing reported the most severe persistent pain, but only if they also had a COMT profile that reflected high pain sensitivity [89]. That finding indicates that genetic influences might moderate the impact of psychosocial factors on pain outcomes. We believe that future studies will identify additional biomarkers, provide validation to existing biomarkers, and elucidate additional pathogenetic mechanisms in CP/CPPS.

\section{POTENTIAL IMPLICATIONS FOR TREATMENT}

Following on the clinical necessity of these findings, a psychotherapy model was proposed that targeted the empirically supported CP/CPPS psychosocial risk factors (depression and catastrophizing) [30], and a study was conducted to examine the treatment feasibility of a risk factor reduction intervention [90]. Specifically, we sought to determine the feasibility of a cognitive-behavioral symptom management program for the acute improvement of psychosocial risk factors of diminished QoL in patients with CP/CPPS. In the study, mean baseline scores showed significant linear reductions in pain, disability, and cat- 
astrophizing over time. In addition, positive but modest changes were made in depressive symptoms and social support. The study also showed that follow-up scores for QoL were improved and $\mathrm{CP} / \mathrm{CPPS}$ symptoms significantly reduced. Furthermore, the baseline to 8-week termination analysis showed that score changes for $\mathrm{CP} / \mathrm{CPPS}$ symptoms and psychosocial risk factors were predicted by reductions in catastrophizing. We concluded that psychosocial management programs can target and significantly reduce several empirically supported psychosocial risk factors associated with poorer CP/CPPS outcomes, but a randomized controlled trial with longitudinal follow-up is needed.

\section{CONCLUSIONS}

The literature on catastrophizing and depression in patients with $\mathrm{CP} / \mathrm{CPPS}$ strongly implicates these factors as having a profound long-term influence on the shaping of pain responses and pain outcomes. To date, abundant evidence suggests that depression and catastrophizing about pain can serve as prospective markers of risk for negative pain-related endpoints, including severe physical disability, disease progression, and lack of improvement following surgical and other interventions. Further research in this area should help to tailor analgesic interventions to individual characteristics, and it seems likely that catastrophizing and depression will be important factors to consider in the selection of individualized treatments. Collectively, catastrophizing and depression are complex biopsychosocial constructs that exert their detrimental effects via multiple overlapping pathways. Fortunately, psychological interventions, particularly cognitivebehavioral therapy, can evidently reduce catastrophizing and depression in some groups of CP/CPPS patients. In the future, it will be important to evaluate the potential long-term benefits of reducing catastrophizing and depression in patients with $\mathrm{CP} /$ CPPS, with a particular focus on studies of tailored early intervention that may help to move patients from a high-risk to a relatively lower risk profile to improve long-term pain outcomes.

\section{CONFLICT OF INTEREST}

No potential conflict of interest relevant to this article was reported.

\section{REFERENCES}

1. Roberts RO, Lieber MM, Rhodes T, Girman CJ, Bostwick DG, Ja- cobsen SJ. Prevalence of a physician-assigned diagnosis of prostatitis: the Olmsted County Study of Urinary Symptoms and Health Status Among Men. Urology 1998;51:578-84.

2. McNaughton Collins M, Meigs JB, Barry MJ, Walker Corkery E, Giovannucci E, Kawachi I. Prevalence and correlates of prostatitis in the health professionals follow-up study cohort. J Urol 2002;167: 1363-6.

3. Woo YN. Prostatitis. Korean J Urol 1994;35:575-85.

4. Krieger JN, Egan KJ, Ross SO, Jacobs R, Berger RE. Chronic pelvic pains represent the most prominent urogenital symptoms of "chronic prostatitis". Urology 1996;48:715-21.

5. Tripp DA, Curtis Nickel J, Landis JR, Wang YL, Knauss JS; CPCRN Study Group. Predictors of quality of life and pain in chronic prostatitis/chronic pelvic pain syndrome: findings from the National Institutes of Health Chronic Prostatitis Cohort Study. BJU Int 2004; 94:1279-82.

6. Davis SN, Maykut CA, Binik YM, Amsel R, Carrier S. Tenderness as measured by pressure pain thresholds extends beyond the pelvis in chronic pelvic pain syndrome in men. J Sex Med 2011;8:232-9.

7. Nickel JC, Roehrborn CG, O’leary MP, Bostwick DG, Somerville MC, Rittmaster RS. Examination of the relationship between symptoms of prostatitis and histological inflammation: baseline data from the REDUCE chemoprevention trial. J Urol 2007;178(3 Pt 1): 896-900.

8. Rudick CN, Berry RE, Johnson JR, Johnston B, Klumpp DJ, Schaeffer AJ, et al. Uropathogenic Escherichia coli induces chronic pelvic pain. Infect Immun 2011;79:628-35.

9. Keltikangas-Jarvinen L, Jarvinen H, Lehtonen T. Psychic disturbances in patients with chronic prostatis. Ann Clin Res 1981;13:45-9.

10. Miller HC. Stress prostatitis. Urology 1988;32:507-10.

11. Reiser MF. Psychophysiology of stress and its sequelae. In: Reiser MF, editor. Mind, brain, body: toward a convergence of psychoanalysis and neurobiology. New York: Basic Books; 1984. p. 161-85.

12. Lazarus RS. Psychological stress and coping in adaptation and illness. Int J Psychiatry Med 1974;5:321-33.

13. Folkman S, Lazarus RS, Dunkel-Schetter C, DeLongis A, Gruen RJ. Dynamics of a stressful encounter: cognitive appraisal, coping, and encounter outcomes. J Pers Soc Psychol 1986;50:992-1003.

14. Ku JH, Kim SW, Paick JS. Quality of life and psychological factors in chronic prostatitis/chronic pelvic pain syndrome. Urology 2005; 66:693-701.

15. Radloff LS. The CES-D scale: a self-report depression scale for research in the general population. Appl Psychol Meas 1977;1:385-401.

16. Egan KJ, Krieger JN. Psychological problems in chronic prostatitis patients with pain. Clin J Pain 1994;10:218-26. 
17. Nickel JC, Tripp DA, Chuai S, Litwin MS, McNaughton-Collins M, Landis JR, et al. Psychosocial variables affect the quality of life of men diagnosed with chronic prostatitis/chronic pelvic pain syndrome. BJU Int 2008;101:59-64.

18. Tripp DA, Nickel JC, Wang Y, Litwin MS, McNaughton-Collins M, Landis JR, et al. Catastrophizing and pain-contingent rest predict patient adjustment in men with chronic prostatitis/chronic pelvic pain syndrome. J Pain 2006;7:697-708.

19. Shoskes DA, Nickel JC, Kattan MW. Phenotypically directed multimodal therapy for chronic prostatitis/chronic pelvic pain syndrome: a prospective study using UPOINT. Urology 2010;75:1249-53.

20. Keogh E, Book K, Thomas J, Giddins G, Eccleston C. Predicting pain and disability in patients with hand fractures: comparing pain anxiety, anxiety sensitivity and pain catastrophizing. Eur J Pain 2010;14:446-51.

21. Sullivan MJ, Thorn B, Haythornthwaite JA, Keefe F, Martin M, Bradley LA, et al. Theoretical perspectives on the relation between catastrophizing and pain. Clin J Pain 2001;17:52-64.

22. Anderson RU, Wise D, Sawyer T, Chan C. Integration of myofascial trigger point release and paradoxical relaxation training treatment of chronic pelvic pain in men. J Urol 2005;174:155-60.

23. Nickel JC, Mullins C, Tripp DA. Development of an evidence-based cognitive behavioral treatment program for men with chronic prostatitis/chronic pelvic pain syndrome. World J Urol 2008;26: 167-72.

24. McNaughton Collins M, Pontari MA, O'Leary MP, Calhoun EA, Santanna J, Landis JR, et al. Quality of life is impaired in men with chronic prostatitis: the Chronic Prostatitis Collaborative Research Network. J Gen Intern Med 2001;16:656-62.

25. Turner JA, Hauge S, Von Korff M, Saunders K, Lowe M, Berger R. Primary care and urology patients with the male pelvic pain syndrome: symptoms and quality of life. J Urol 2002;167:1768-73.

26. Clemens JQ, Brown SO, Calhoun EA. Mental health diagnoses in patients with interstitial cystitis/painful bladder syndrome and chronic prostatitis/chronic pelvic pain syndrome: a case/control study. J Urol 2008;180:1378-82.

27. Chung SD, Huang CC, Lin HC. Chronic prostatitis and depressive disorder: a three year population-based study. J Affect Disord 2011;134:404-9.

28. Ahn SG, Kim SH, Chung KI, Park KS, Cho SY, Kim HW. Depression, anxiety, stress perception, and coping strategies in korean military patients with chronic prostatitis/chronic pelvic pain syndrome. Korean J Urol 2012;53:643-8.

29. Tripp DA, Nickel JC, Ross S, Mullins C, Stechyson N. Prevalence, symptom impact and predictors of chronic prostatitis-like symp- toms in Canadian males aged 16-19 years. BJU Int 2009;103:1080-4.

30. Shoskes DA, Nickel JC, Rackley RR, Pontari MA. Clinical phenotyping in chronic prostatitis/chronic pelvic pain syndrome and interstitial cystitis: a management strategy for urologic chronic pelvic pain syndromes. Prostate Cancer Prostatic Dis 2009;12:177-83.

31. Nickel JC, Downey J, Ardern D, Clark J, Nickel K. Failure of a monotherapy strategy for difficult chronic prostatitis/chronic pelvic pain syndrome. J Urol 2004;172:551-4.

32. Nickel JC, Downey JA, Nickel KR, Clark JM. Prostatitis-like symptoms: one year later. BJU Int 2002;90:678-81.

33. Krieger JN, Nyberg L Jr, Nickel JC. NIH consensus definition and classification of prostatitis. JAMA 1999;282:236-7.

34. Schaeffer AJ, Datta NS, Fowler JE Jr, Krieger JN, Litwin MS, Nadler $\mathrm{RB}$, et al. Overview summary statement. Diagnosis and management of chronic prostatitis/chronic pelvic pain syndrome (CP/CPPS). Urology 2002;60(6 Suppl):1-4.

35. Deyo RA, Weinstein JN. Low back pain. N Engl J Med 2001;344: 363-70.

36. Blaivas JG, Weiss JP, Desai P, Flisser AJ, Stember DS, Stahl PJ. Longterm followup of augmentation enterocystoplasty and continent diversion in patients with benign disease. J Urol 2005;173:1631-4.

37. Flood HD, Malhotra SJ, O'Connell HE, Ritchey MJ, Bloom DA, McGuire EJ. Long-term results and complications using augmentation cystoplasty in reconstructive urology. Neurourol Urodyn 1995; 14:297-309.

38. Nickel JC. Chronic prostatitis/chronic pelvic pain: the syndrome. J Urol 2009;182:18-9.

39. Nickel JC. Words of wisdom. Re: Clinical phenotyping in chronic prostatitis/chronic pelvic pain syndrome and interstitial cystitis: a management strategy for urologic chronic pelvic pain syndromes. Eur Urol 2009;56:881.

40. Tripp DA, Nickel JC, Shoskes D, Koljuskov A. A 2-year follow-up of quality of life, pain, and psychosocial factors in patients with chronic prostatitis/chronic pelvic pain syndrome and their spouses. World J Urol 2013 Apr 9 [Epub]. http://dx.doi.org/10.1007/s00345013-1067-6.

41. Wenninger K, Heiman JR, Rothman I, Berghuis JP, Berger RE. Sickness impact of chronic nonbacterial prostatitis and its correlates. J Urol 1996;155:965-8.

42. Rodríguez MA, Afari N, Buchwald DS; National Institute of Diabetes and Digestive and Kidney Diseases Working Group on Urological Chronic Pelvic Pain. Evidence for overlap between urological and nonurological unexplained clinical conditions. J Urol 2009;182: 2123-31.

43. Litwin MS, McNaughton-Collins M, Fowler FJ Jr, Nickel JC, Cal- 
houn EA, Pontari MA, et al. The National Institutes of Health chronic prostatitis symptom index: development and validation of a new outcome measure. Chronic Prostatitis Collaborative Research Network. J Urol 1999;162:369-75.

44. Alexander RB, Propert KJ, Schaeffer AJ, Landis JR, Nickel JC, O'Leary MP, et al. Ciprofloxacin or tamsulosin in men with chronic prostatitis/chronic pelvic pain syndrome: a randomized, double-blind trial. Ann Intern Med 2004;141:581-9.

45. Dimitrakov J, Guthrie D. Genetics and phenotyping of urological chronic pelvic pain syndrome. J Urol 2009;181:1550-7.

46. MAPP Research Network. A New Look at Urological Chronic Pelvic Pain [Internet]. Philadelphia: University of Pennsylvania; 2008 [cited 2013 jun 10]. Available from: http://www.mappnetwork.org/

47. Nickel JC, Shoskes D. Phenotypic approach to the management of chronic prostatitis/chronic pelvic pain syndrome. Curr Urol Rep 2009;10:307-12.

48. Bernal RM, Pontari MA. Evaluation of chronic pelvic pain syndrome in men: is it chronic prostatitis? Curr Urol Rep 2009;10:295-301.

49. Allsop SA, Erstad DJ, Brook K, Bhai SF, Cohen JM, Dimitrakoff JD. The DABBEC phenotyping system: towards a mechanistic understanding of CP/CPPS. Nat Rev Urol 2011;8:107-13.

50. Nickel JC. Clinical evaluation of the man with chronic prostatitis/ chronic pelvic pain syndrome. Urology 2002;60(6 Suppl):20-2.

51. Staab JP, Datto CJ, Weinrieb RM, Gariti P, Rynn M, Evans DL. Detection and diagnosis of psychiatric disorders in primary medical care settings. Med Clin North Am 2001;85:579-96.

52. Osman A, Barrios FX, Kopper BA, Hauptmann W, Jones J, O’Neill E. Factor structure, reliability, and validity of the Pain Catastrophizing Scale. J Behav Med 1997;20:589-605.

53. Briggs EL, Sullivan DJ, Bernholc J. Large-scale electronic-structure calculations with multigrid acceleration. Phys Rev B Condens Matter 1995;52:R5471-4.

54. Anderson RU, Orenberg EK, Morey A, Chavez N, Chan CA. Stress induced hypothalamus-pituitary-adrenal axis responses and disturbances in psychological profiles in men with chronic prostatitis/ chronic pelvic pain syndrome. J Urol 2009;182:2319-24.

55. Berghuis JP, Heiman JR, Rothman I, Berger RE. Psychological and physical factors involved in chronic idiopathic prostatitis. J Psychosom Res 1996;41:313-25.

56. de la Rosette JJ, Ruijgrok MC, Jeuken JM, Karthaus HF, Debruyne FM. Personality variables involved in chronic prostatitis. Urology 1993;42:654-62.

57. Mehik A, Hellstrom P, Sarpola A, Lukkarinen O, Jarvelin MR. Fears, sexual disturbances and personality features in men with prostatitis: a population-based cross-sectional study in Finland. BJU Int
2001;88:35-8.

58. Anderson RU. Commentary on the mechanisms in prostatitis/ chronic pelvic pain syndrome. J Urol 2008;179(5 Suppl):S68.

59. Sullivan MJ, Stanish W, Sullivan ME, Tripp D. Differential predictors of pain and disability in patients with whiplash injuries. Pain Res Manag 2002;7:68-74.

60. Gatenbeck L, Aronsson A, Dahlgren S, Johansson B, Stromberg L. Stress stimuli-induced histopathological changes in the prostate: an experimental study in the rat. Prostate 1987;11:69-76.

61. Spanos C, Pang X, Ligris K, Letourneau R, Alferes L, Alexacos N, et al. Stress-induced bladder mast cell activation: implications for interstitial cystitis. J Urol 1997;157:669-72.

62. Miller LJ, Fischer KA, Goralnick SJ, Litt M, Burleson JA, Albertsen P, et al. Nerve growth factor and chronic prostatitis/chronic pelvic pain syndrome. Urology 2002;59:603-8.

63. Borish L, Schmaling K, DiClementi JD, Streib J, Negri J, Jones JF. Chronic fatigue syndrome: identification of distinct subgroups on the basis of allergy and psychologic variables. J Allergy Clin Immunol 1998;102:222-30.

64. Pontari MA, Ruggieri MR. Mechanisms in prostatitis/chronic pelvic pain syndrome. J Urol 2008;179(5 Suppl):S61-7.

65. Campbell CM, Edwards RR. Mind-body interactions in pain: the neurophysiology of anxious and catastrophic pain-related thoughts. Transl Res 2009;153:97-101.

66. Gracely RH, Geisser ME, Giesecke T, Grant MA, Petzke F, Williams DA, et al. Pain catastrophizing and neural responses to pain among persons with fibromyalgia. Brain 2004;127(Pt 4):835-43.

67. Farmer MA, Chanda ML, Parks EL, Baliki MN, Apkarian AV, Schaeffer AJ. Brain functional and anatomical changes in chronic prostatitis/chronic pelvic pain syndrome. J Urol 2011;186:117-24.

68. Strigo IA, Simmons AN, Matthews SC, Craig AD, Paulus MP. Increased affective bias revealed using experimental graded heat stimuli in young depressed adults: evidence of "emotional allodynia". Psychosom Med 2008;70:338-44.

69. Berna C, Leknes S, Holmes EA, Edwards RR, Goodwin GM, Tracey I. Induction of depressed mood disrupts emotion regulation neurocircuitry and enhances pain unpleasantness. Biol Psychiatry 2010;67:1083-90.

70. Mordasini L, Weisstanner C, Rummel C, Thalmann GN, Verma $\mathrm{RK}$, Wiest R, et al. Chronic pelvic pain syndrome in men is associated with reduction of relative gray matter volume in the anterior cingulate cortex compared to healthy controls. J Urol 2012;188: 2233-7.

71. Davis KD, Pope G, Chen J, Kwan CL, Crawley AP, Diamant NE. Cortical thinning in IBS: implications for homeostatic, attention, 
and pain processing. Neurology 2008;70:153-4.

72. May A. Chronic pain may change the structure of the brain. Pain 2008;137:7-15.

73. Schmidt-Wilcke T, Ganssbauer S, Neuner T, Bogdahn U, May A. Subtle grey matter changes between migraine patients and healthy controls. Cephalalgia 2008;28:1-4.

74. Hsu MC, Harris RE, Sundgren PC, Welsh RC, Fernandes CR, Clauw DJ, et al. No consistent difference in gray matter volume between individuals with fibromyalgia and age-matched healthy subjects when controlling for affective disorder. Pain 2009;143:262-7.

75. Schmidt-Wilcke T, Leinisch E, Ganssbauer S, Draganski B, Bogdahn $\mathrm{U}$, Altmeppen J, et al. Affective components and intensity of pain correlate with structural differences in gray matter in chronic back pain patients. Pain 2006;125:89-97.

76. Lutgendorf SK, Kreder KJ, Rothrock NE, Hoffman A, Kirschbaum C, Sternberg EM, et al. Diurnal cortisol variations and symptoms in patients with interstitial cystitis. J Urol 2002;167:1338-43.

77. Crofford LJ, Pillemer SR, Kalogeras KT, Cash JM, Michelson D, Kling MA, et al. Hypothalamic-pituitary-adrenal axis perturbations in patients with fibromyalgia. Arthritis Rheum 1994;37:1583-92.

78. Geiss A, Varadi E, Steinbach K, Bauer HW, Anton F. Psychoneuroimmunological correlates of persisting sciatic pain in patients who underwent discectomy. Neurosci Lett 1997;237:65-8.

79. Pruessner JC, Hellhammer DH, Kirschbaum C. Burnout, perceived stress, and cortisol responses to awakening. Psychosom Med 1999; 61:197-204.

80. Schulz P, Kirschbaum C, Prußner J, Hellhammer D. Increased free cortisol secretion after awakening in chronically stressed individuals due to work overload. Stress Med 1998;14:91-7.

81. Wust S, Federenko I, Hellhammer DH, Kirschbaum C. Genetic factors, perceived chronic stress, and the free cortisol response to awakening. Psychoneuroendocrinology 2000;25:707-20.

82. Pruessner M, Hellhammer DH, Pruessner JC, Lupien SJ. Self-re- ported depressive symptoms and stress levels in healthy young men: associations with the cortisol response to awakening. Psychosom Med 2003;65:92-9.

83. Weissman MM, Fyer AJ, Haghighi F, Heiman G, Deng Z, Hen R, et al. Potential panic disorder syndrome: clinical and genetic linkage evidence. Am J Med Genet 2000;96:24-35.

84. Weissman MM, Gross R, Fyer A, Heiman GA, Gameroff MJ, Hodge SE, et al. Interstitial cystitis and panic disorder: a potential genetic syndrome. Arch Gen Psychiatry 2004;61:273-9.

85. Blok BF. Central pathways controlling micturition and urinary continence. Urology 2002;59(5 Suppl 1):13-7.

86. Cohen H, Neumann L, Glazer Y, Ebstein RP, Buskila D. The relationship between a common catechol-O-methyltransferase (COMT) polymorphism val(158) met and fibromyalgia. Clin Exp Rheumatol 2009;27(5 Suppl 56):S51-6.

87. Fijal B, Perlis RH, Heinloth AN, Houston JP. The association of single nucleotide polymorphisms in the catechol-O-methyltransferase gene and pain scores in female patients with major depressive disorder. J Pain 2010;11:910-5, 915.e1-9.

88. Diatchenko L, Slade GD, Nackley AG, Bhalang K, Sigurdsson A, Belfer I, et al. Genetic basis for individual variations in pain perception and the development of a chronic pain condition. Hum Mol Genet 2005;14:135-43.

89. George SZ, Wallace MR, Wright TW, Moser MW, Greenfield WH 3rd, Sack BK, et al. Evidence for a biopsychosocial influence on shoulder pain: pain catastrophizing and catechol-O-methyltransferase (COMT) diplotype predict clinical pain ratings. Pain 2008; 136:53-61.

90. Tripp DA, Nickel JC, Katz L. A feasibility trial of a cognitive-behavioural symptom management program for chronic pelvic pain for men with refractory chronic prostatitis/chronic pelvic pain syndrome. Can Urol Assoc J 2011;5:328-32. 